Original article

\title{
Mechanical effects of residual bone mass, a maxillofacial prosthesis, and a reconstruction plate on the mandible after marginal resection
}

\author{
Division of Applied Oral Sciences, Nihon University Graduate School of Dentistry, Tokyo, Japan \\ 2) Department of Partial Denture Prosthodontics, Nihon University School of Dentistry, Tokyo, Japan \\ ${ }^{3)}$ Division of Clinical Research, Dental Research Center, Nihon University School of Dentistry, Tokyo, Japan \\ 4) Department of Dental Materials, Nihon University School of Dentistry, Tokyo, Japan \\ ${ }^{5)}$ Division of Biomaterial Science, Dental Research Center, Nihon University School of Dentistry, Tokyo, Japan
}

Takeshi Kase ${ }^{1)}$, Tetsuo Ohyama ${ }^{2,3)}$, Shinya Nakabayashi ${ }^{2,3)}$, Hiroyasu Yasuda ${ }^{2,3)}$, and Takayuki Yoneyama ${ }^{4,5}$

(Received October 11, 2019; Accepted December 14, 2019)

\begin{abstract}
In this study, analytic models were used to simulate marginal resection in the area of the second premolar to the second molar region, and the mechanical effects on the mandible of residual bone mass, a maxillofacial prosthesis, and a reconstruction plate were evaluated by three-dimensional finite element analysis. As residual bone mass decreased, maximum principal stress increased near the anterior ramus of the mandible, and maximum shear stress increased at the anterior buccal region of the resected area. In the mandible with a maxillofacial prosthesis, the maximum principal stress distribution at the anterior ramus was lower, and the distribution of maximum shear stress at the anterior buccal region of the resected area was higher. When a reconstruction plate was used, maximum principal stress and maximum shear stress were lower. Thus, lower residual bone mass was associated with increased mandible deflection and torsion. In addition, presence of a maxillofacial prosthesis decreased deflection but increased torsion, and presence of a reconstruction plate decreased deflection and greatly decreased torsion. These findings suggest that decreased residual bone mass and maxillofacial prostheses increase fracture risk; however, presence of a reconstruction plate was effective in decreasing torsional stress, thereby reducing fracture risk in the mandible.
\end{abstract}

Keywords; finite element analysis, marginal resection, maxillofacial prostheses, reconstruction plate

\section{Introduction}

Marginal resection of the mandible is a tumor surgery that preserves the inferior border of the mandible and minimizes postoperative morphological and functional deformities of the mandible [1]. Maxillofacial prosthetic treatment is required after marginal resection of the mandible [2]. The objectives of maxillofacial prostheses are restoration of maxillofacial morphology and masticatory function, improvement of esthetics and mental wellbeing, and support of patient social reintegration and quality of life [3]. However, bone fracture sometimes occurs because of bone thinning and diminished bone strength after marginal resection [4].

Fracture risk is higher if the thickness of the residual mandible after marginal resection is less than $10 \mathrm{~mm}$ [5]. Okuyama et al. reported that strong occlusal force was a significant risk factor and that that the risk of mandibular fracture was higher for patients with maxillofacial prostheses than for those without such prostheses [6]. However, no studies have evaluated dynamically the association between maxillofacial prostheses and mandibular fracture, and the details are thus unclear. To prevent fracture after marginal resection, mandibular reconstruction plates are often used for the remaining mandible. This method has been widely used clinically and is effective [7]; however, little is known of the mechanical effects of residual mandibular bone mass, reconstruction plates, and maxillofacial

Correspondence to Dr. Tetsuo Ohyama, Department of Partial Denture Prosthodontics, Nihon University School of Dentistry, 1-8-13 Kanda-Surugadai, Chiyoda-ku, Tokyo 101-8310, Japan Fax:+81-3-3219-8350 E-mail: ohyama.tetsuo@nihon-u.ac.jp

J-STAGE Advance Publication: August 1, 2020

Color figures can be viewed in the online issue at J-STAGE.

doi.org/10.2334/josnusd.19-0427

DN/JST.JSTAGE/josnusd/19-0427 prostheses on cortical bone. This study used three-dimensional finite element analysis to evaluate the mechanical effects of residual mandibular bone mass, a maxillofacial prosthesis, and a reconstruction plate on cortical bone after marginal resection of the mandible.

\section{Materials and Methods}

\section{Construction of three-dimensional finite element models}

The analytic models included cancellous bone, cortical bone, residual ridge mucosa, teeth, periodontal ligament, denture base resin, a metal frame, and a titanium reconstruction plate and screws. Models without a left mandibular second premolar, without first and second molars, and with a mandible with no defects were constructed, as were models with marginal resection of the area of the second premolar to the second molar region. The mandibular models used in this study are shown in Fig. 1. Model I had no defects, Model II was resected along the mylohyoid line, and Model III was resected along the inferior border of the mandibular canal. Model ID was Model I with a removable partial denture, Models IID and IIID were Models II and III, respectively, with a maxillofacial prosthesis, and Models IIIP and IIIPD were Models III and IIID, respectively, with a reconstruction plate at the area of the defect. To avoid concentration of stress, a curve was made at the corner of the resected area [8]. In the prosthetic design, the RPI clasp was applied to the left first premolar (as the direct retainer), the mesial rest was applied to the right first premolar, and the Akers clasp was fastened to the right first molar (Fig. 2). The reconstruction plate was used at the inferior border of the mandibular body and fixed with screws at both ends. Model I comprised 293,036 elements and 531,652 nodes, Model ID had 303,279 elements and 551,418 nodes, Model II included 374,260 elements and 676,690 nodes, Model IID comprised 389,900 elements and 705,947 nodes, Model III had 327,721 elements and 591,015 nodes, Model IIID included 341,102 elements and 616,489 nodes, Model IIIP comprised 330,442 elements and 596,529 nodes, and Model IIIPD had 347,062 elements and 627,556 nodes.

The analytic model for the mandible was created by injecting epoxy resin (Devcon ET, Fujikura Ohyo-Kako, Kawaguchi, Japan) into the impression of a skull replica model (P10-SB1, Nissin Japan, Ltd., Kyoto, Japan) made with an impression material (Duplicone, Shofu Inc., Kyoto, Japan), in accordance with the manufacturer's instructions. The mold was imaged with a multidetector CT device (AsteionTM Super4 Edition, Toshiba, Tokyo, Japan). On the basis of these imaging data, mandibular contours were constructed with an all-purpose computer-aided design program (Rhinoceros Ver. 1.0, Robert McNeel \& Associates, Seattle, WA, USA), an all-purpose finite element analysis program (ANSYS Mechanical Rel.17.0, ANSYS, Inc., Canonsburg, PA, USA), and a 3D direct modeler (ANSYS SpaceClaim Direct Modeler, SpaceClaim Corp., Canonsburg, PA, USA). The dimensions of cortical bone, cancellous bone, and residual ridge mucosa were constructed by using information from previous reports [9-11]. In the contact condition, the denture base resin and metal frame against the residual ridge mucosa and teeth were set as contact elements.

Table 1 shows the material property values of cortical bone [12], cancellous bone [13], teeth [14], residual ridge mucosa [15], denture base resin [16], the metal framework [17], and the titanium plate and screws [18], which were set by using previously reported information. The mate- 

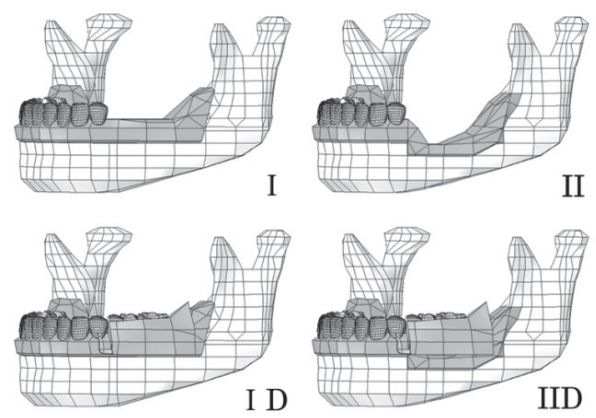

Fig. 1 Analytic models

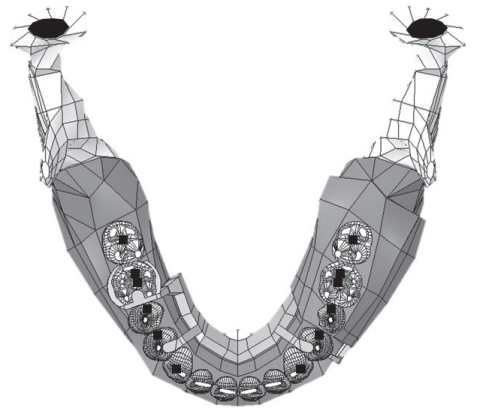

Fig. 2 Prosthesis design and constraint conditions

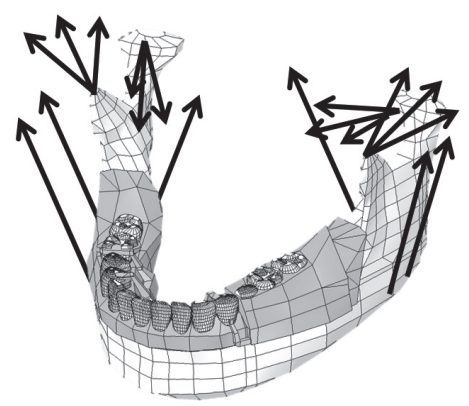

Muscle vector :<smiles>[Y]C([Y])[X]</smiles>

Fig. 3 Loading conditions

rial property values of the periodontal ligament were set so that the load displacement characteristics were similar to actual in vivo values $[19,20]$.

\section{Loading conditions}

The loading conditions (Fig. 3; Table 2) and were set to simulate muscle contraction during clenching in the premolar and molar regions. The direction and amount of contraction of each muscle were calculated after being converted into vectors for overall coordinates (X, Y, Z) [21]. These values were applied to the equivalent attachment area at the shallow and deep regions of the masseter muscle, the medial pterygoid muscle, the anterior, central, and posterior regions of the temporal muscle, and the inferior and superior regions of the lateral pterygoid muscle and the anterior belly of digastric muscle.

\section{Constraint conditions}

The constraint conditions are shown in Fig. 2. The top of the condylar heads and occlusal contact points with their maxillary antagonists were defined as constraint conditions in the $\mathrm{X}, \mathrm{Y}$, and $\mathrm{Z}$ directions. The occlusal contact points were set at the mandibular canine tips, the distal fossae of first and second premolars, the central and distal fossae of first molars, and the central fossae of second molars [21].

\section{Analysis items}

Stress was evaluated by measuring maximum principal stress and maximum shear stress at the mandibular left-side second premolar, first molar,
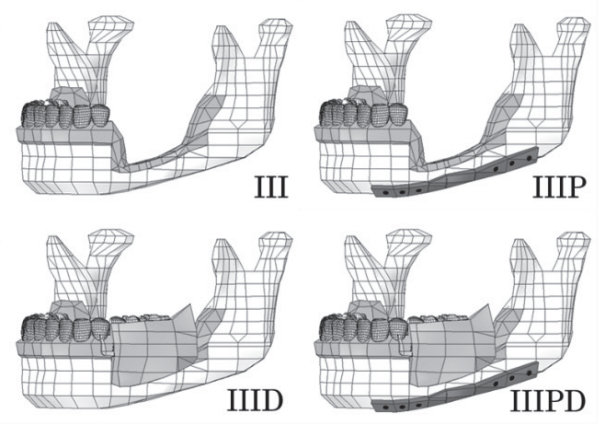

Table 1 Material properties used in this study

\begin{tabular}{lcc}
\hline Model & Young's modulus $(\mathrm{MPa})$ & Poisson's ratio \\
\hline Cortical bone & $2.28 \times 10^{4}$ & 0.30 \\
Cancellous bone & $7.80 \times 10^{3}$ & 0.30 \\
Teeth & $1.37 \times 10^{4}$ & 0.30 \\
Residual ridge mucosa & $4.50 \times 10^{-2}$ & 0.49 \\
Denture base resin & $1.96 \times 10^{3}$ & 0.33 \\
Metal framework & $2.00 \times 10^{5}$ & 0.33 \\
Titanium plate and screws & $1.10 \times 10^{5}$ & 0.33 \\
Periodontal ligament (first load) & $4.90 \times 10^{-2}$ & 0.49 \\
Periodontal ligament (second load) & $0.30 \times 10$ & 0.49 \\
\hline
\end{tabular}

Table 2 Loading forces used in this study

\begin{tabular}{llcc}
\hline & & Number of nodes & Loading force $(\mathrm{N})$ \\
\hline Masseter muscle & Shallow & 14 & 190.4 \\
& Deep & 5 & 81.6 \\
Medial pterygoid muscle & & 11 & 132.8 \\
Temporal muscle & Anterior & 9 & 154.8 \\
& Central & 12 & 91.8 \\
& Posterior & 9 & 71.1 \\
Lateral pterygoid muscle & Inferior & 3 & 18.1 \\
& Superior & 3 & 17.0 \\
Digastric muscle & Anterior belly & 1 & 11.2 \\
\hline
\end{tabular}

and second molar regions.

\section{Results}

Mechanical effects of residual mandibular bone mass, the removable partial denture, and the maxillofacial prosthesis on cortical bone

Figure 4 shows the distributions of maximum principal stress in Models I, ID, II, IID, III, and IIID. Maximum principal stress was concentrated near the anterior ramus of the mandible, and the ranking of the distribution of maximum principal stress, in ascending order, was I, II, III. Stress concentration was markedly diminished in models with the removable partial denture and maxillofacial prosthesis.

Figure 5 shows maximum principal stress values. The ranking of maximum principal stress, in descending order, was III, II, I. Maximum principal stress values were $13 \%$ to $16 \%$ lower for models with the removable partial denture and maxillofacial prosthesis.

Figure 6 shows the distributions of maximum shear stress in Models I, ID, II, IID, III, and IIID. Maximum shear stress was concentrated near the anterior ramus of the mandible in I and at the anterior buccal region of the resected area in II and III. The ranking of the distribution of maximum shear stress, in ascending order, was I, II, III. In models with the removable partial denture and maxillofacial prosthesis (ID, IID, and IIID) the stress distribution near the anterior ramus of the mandible was lower. However, stress and stress distribution were greater at the anterior buccal region of the resected area in Models II and III. 


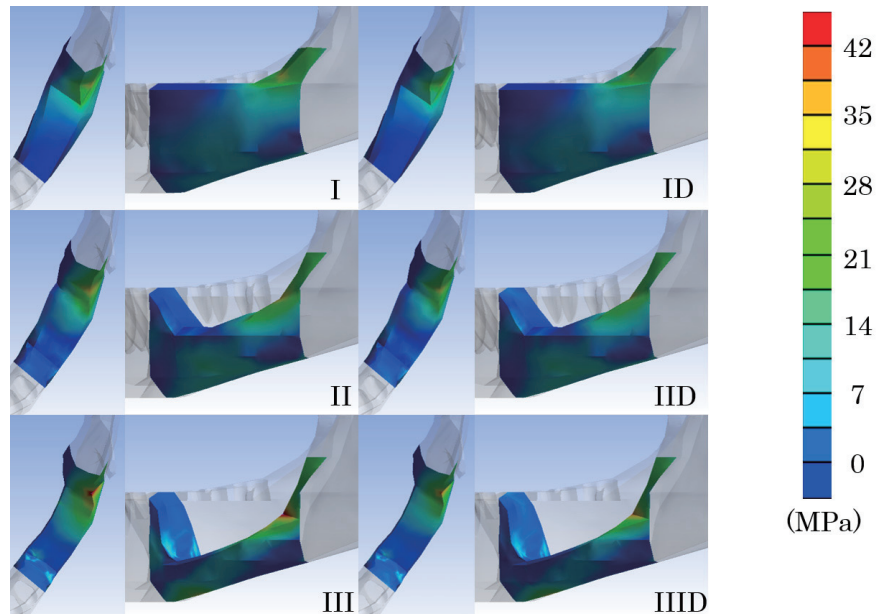

Fig. 4 Distribution of maximum principal stress values for cortical bone in models including residual mandibular bone mass, a removable partial denture, and a maxillofacial prosthesis

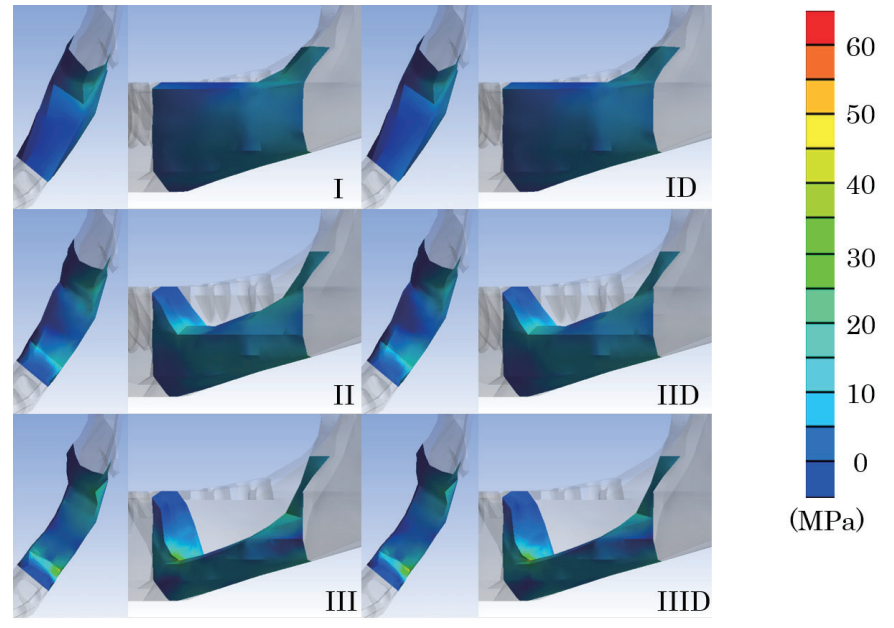

Fig. 6 Distribution of maximum shear stress values for cortical bone in models including residual mandibular bone mass, a removable partial denture, and a maxillofacial prosthesis

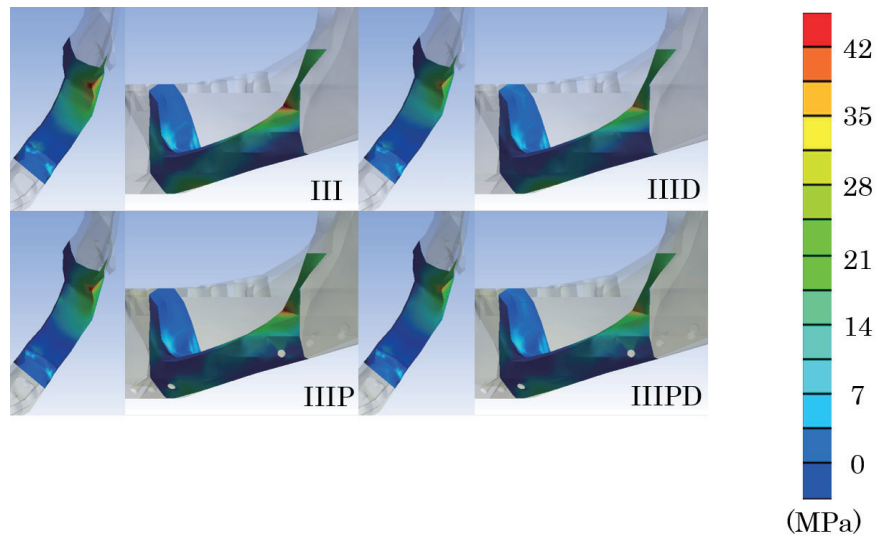

Fig. 8 Distribution of maximum principal stress values for cortical bone in models including a reconstruction plate

Figure 7 shows maximum shear stress values. The ranking of maximum shear stress, in descending order, was III, II, I. In models with the removable partial denture and maxillofacial prosthesis, maximum shear stress values were $15 \%$ lower in I and $9 \%$ higher in II and III.

\section{Effect of the reconstruction plate on cortical bone}

Figure 8 shows distributions of maximum principal stress in Models III, IIID, IIIP, and IIIPD. In comparisons between III and IIIP and between

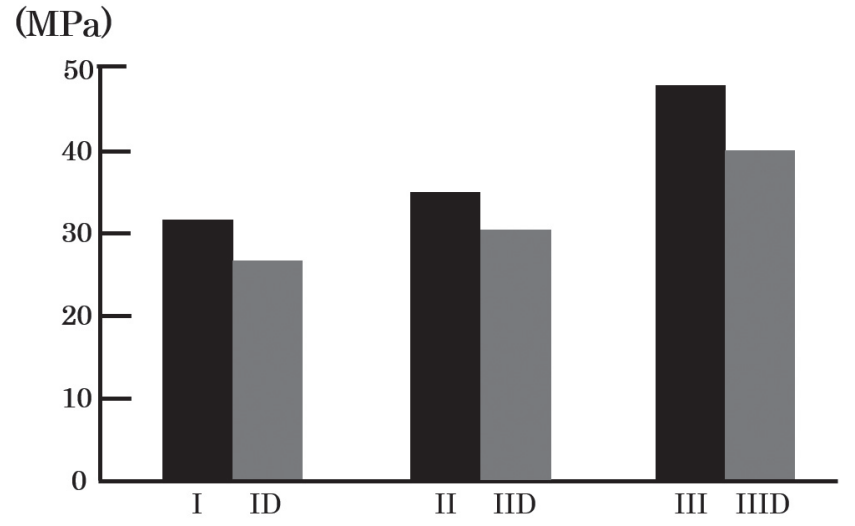

Fig. 5 Maximum principal stress values for cortical bone in models including residual mandibular bone mass, a removable partial denture, and a maxillofacial prosthesis

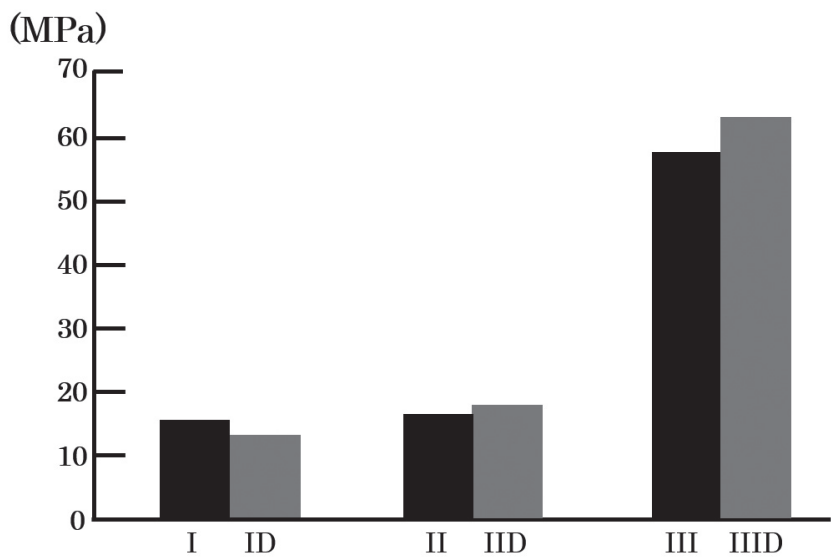

Fig. 7 Maximum shear stress values for cortical bone in models including residual mandibular bone mass, a removable partial denture, and a maxillofacial prosthesis

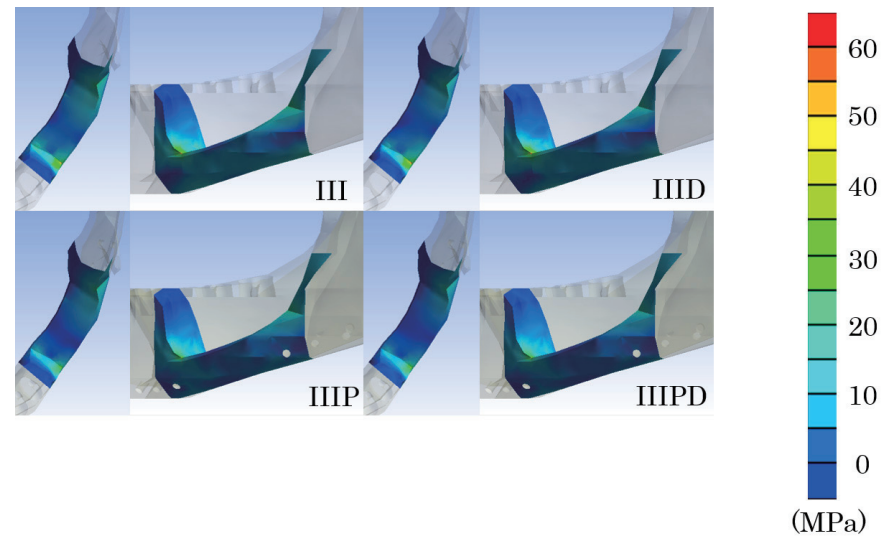

Fig. 9 Distribution of maximum shear stress values for cortical bone in models including a reconstruction plate

IIID and IIIPD, presence of a reconstruction plate was associated with lower maximum principal stress values.

Figure 9 shows distributions of maximum shear stress in Models III, IIID, IIIP, and IIIPD. In comparisons between III and IIIP and between IIID and IIIPD, presence of a reconstruction plate was associated with lower maximum shear stress values.

Figures 10 and 11 show maximum principal stress and shear stress values. In comparisons between III and IIIP and between IIID and IIIPD, 


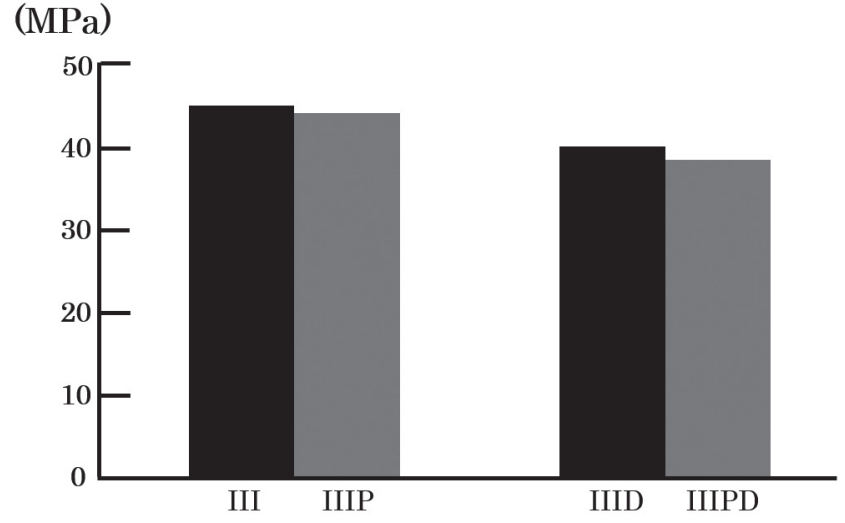

Fig. 10 Maximum principal stress values for cortical bone in models including a reconstruction plate

presence of a reconstruction plate decreased maximum principal stress and maximum shear stress values. Maximum principal stress was $4 \%$ to $8 \%$ lower, and maximum shear stress was $24 \%$ to $27 \%$ lower.

\section{Discussion}

Finite element analysis has recently been utilized in dental research. This method uses contour diagrams to illustrate quantitatively the distribution of stress applied to an object, thereby clarifying its mechanical characteristics. In cases of prosthetic treatment with an implant-assisted removable partial denture for a missing mandibular unilateral distal extension, the mechanical effects of implant position and implant abutment height on the abutment tooth, denture, and tissue supporting the denture were evaluated with three-dimensional finite element analysis [22]. Because the present study used a three-dimensional model to examine mandible deflection and torsion, three-dimensional finite element analysis was selected.

The mandible is loaded by deflection and torsion [23] and, to better simulate conditions in the human body, the present analysis considered muscle contraction during clenching. During contraction of muscles of mastication, deflection and torsion occur in the mandible. Mandibular deflection causes tensile and compressive stress, and torsion results in shear stress. Bone strength depends on tension and shear stress [24]. Numerous studies have used tensile stress and maximum shear stress to evaluate fracture risk, and maximum principal stress and maximum shear stress were used to evaluate stress in this study.

Maximum principal stress was concentrated near the anterior ramus of the mandible. Expansion of the distribution of maximum principal stress was confirmed, and the ranking of maximum principal stress was, in ascending order, I, II, III. Maximum shear stress was concentrated near the anterior ramus of the mandible in I and at the anterior buccal region of the resected area in II and III. The ranking of the distribution of maximum shear stress and maximum shear stress values, in ascending order, was I, II, III. These findings appear to reflect a change in biomechanical behavior caused by increased deflection and shear in the mandible resulting from muscle contraction during occlusion, as residual mandibular bone mass and bone strength decrease.

In models of cortical bone with the removable partial denture and maxillofacial prosthesis, the distribution of maximum principal stress near the anterior ramus of the mandible was lower, and the distribution of maximum shear stress at the anterior buccal part of the resected area was higher. Maximum principal and shear stress values changed because occlusal contact points were increased by the presence of the denture and maxillofacial prosthesis, which suppressed upward displacement of the mandible during muscle contraction and increased outward rotation. Thus, maximum principal stress was decreased by limiting upward displacement and decreasing deflection of the mandible, and maximum shear stress was augmented by increasing outward rotation and increasing shear of the mandible. Because the maximum shear stress value was $57.7 \mathrm{MPa}$ for III and 63.4 MPa for IIID, and the shear strength of bone is 65 to $71 \mathrm{MPa}$ [24], presence of a maxillofacial prosthesis may increase fracture risk.

Analysis of maximum shear stress revealed a different trend for ID

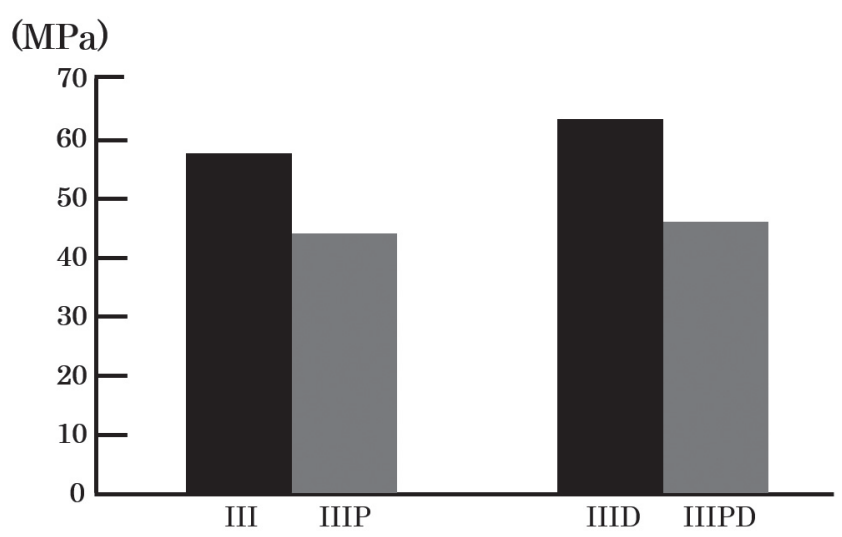

Fig. 11 Maximum shear stress values for cortical bone in models including a reconstruction plate

than for IID and IIID. Maximum shear stress was concentrated near the anterior ramus of the mandible in ID but not in IID and IIID. Because the anterior ramus of the mandible was not affected by outward rotation of the mandible with increasing occlusal contact points, this finding may be attributable to stabilization by the denture.

In the model of cortical bone with the reconstruction plate, the distribution of maximum principal stress was lower near the anterior ramus of the mandible, and the distribution of maximum shear stress was lower at the anterior buccal region of the resected area. These changes in maximum principal and maximum shear stress reflect differences in the biomechanical behavior of the mandible and are caused by the presence of a reconstruction plate and reduction of deflection and torsion in the mandible during clenching. Maximum principal stress decreased by $4 \%$ to $8 \%$ and maximum shear stress decreased by $24 \%$ to $27 \%$; thus, the reconstruction plate appears to be useful for decreasing torsion. Reconstruction plates help prevent mandibular fracture, as stress is markedly lower in the resected area because deflection is decreased and torsion is greatly reduced in the mandible [25].

In sum, the present findings suggest that decreased residual mandibular bone mass and the presence of a removable maxillofacial prosthesis increase fracture risk. However, the presence of a reconstruction plate reinforced the mandible, suppressed torsion, and reduced fracture risk. The present model of marginal resection at the area of the second premolar to the second molar, along the inferior border of the mandibular canal, with a maxillofacial prosthesis, exhibited torsion and higher fracture risk. However, the presence of a reconstruction plate limited torsional stress and thus might help prevent mandibular fracture.

\section{Acknowledgments}

This research was supported by a grant from the Dental Research Center of Nihon University School of Dentistry.

\section{Conflict of interest}

The authors declare no conflict of interest.

\section{References}

1. Beecroft WA, Sako K, Razack MS, Shedd DP (1982) Mandible preservation in the treatment of cancer of the floor of the mouth. J Surg Oncol 19, 171-175.

2. Ferreira AN, Aras M, Chitre V, Mascarenhas K (2018) Prosthetic rehabilitation of a patient with ameloblastoma using an unconventional cast partial denture. J Dent App 5, 435-437.

3. Hagio M, Ishizaki K, Ryu M, Nomura T, Takano N, Sakurai K (2018) Maxillofacial prosthetic treatment factors affecting oral health-related quality of life after surgery for patients with oral cancer. J Prosthet Dent 119, 663-670.

4. Murakami K, Sugiura T, Yamamoto K, Kawakami M, Kang YB, Tsutsumi S et al. (2011) Biomechanical analysis of the strength of the mandible after marginal resection. J Oral Maxillofac Surg 69, 1798-1806.

5. Barttelbort SW, Ariyan S (1993) Mandible preservation with oral cavity carcinoma: rim mandibulectomy versus sagittal mandibulectomy. Am J Surg 166, 411-415.

6. Okuyama K, Michi Y, Mizutani M, Yamashiro M, Kaida A, Harada K (2016) Clinical study on mandibular fracture after marginal resection of the mandible. Oral Surg Oral Med Oral Pathol Oral Radiol 121, 461-467.

7. Cohen A, Laviv A, Berman P, Nashef R, Abu-Tair J (2009) Mandibular reconstruction using stereolithographic 3-dimensional printing modeling technology. Oral Surg Oral Med Oral Pathol Oral Radiol Endod 108, 661-666. 
8. Wittkampf AR, Starmans FJ (1995) Prevention of mandibular fractures by using constructional design principles. I. Computer simulation of human mandibular strength after segmental resections. Int J Oral Maxillofac Surg 24, 306-310.

9. Dong J, Zhang FY, Wu GH, Zhang W, Yin J (2015) Measurement of mucosal thickness in denture-bearing area of edentulous mandible. Chin Med J 128, 342-347.

10. Nucera R, Lo Gludice A, Bellocchio AM, Spinuzza P, Caprioglio A, Perillo L et al. (2017) Bone and cortical bone thickness of mandibular buccal shelf for mini-screw insertion in adults. Angle Orthod 87, 745-751.

11. Promma L, Sakulsak N, Putiwat P, Amarttayakong P, Iamsaard S, Trakulsuk H et al. (2017) Cortical bone thickness of the mandibular canal and implications for bilateral sagittal split osteotomy: a cadaveric study. Int J Oral Maxillofac Surg 46, 572-577.

12. Mente PL, Lewis JL (1989) Experimental method for the measurement of the elastic modulus of trabecular bone tissue. J Orthop Res 7, 456-461.

13. Schwartz-Dabney CL, Dechow PC (2003) Variations in cortical material properties throughout the human dentate mandible. Am J Phys Anthropol 120, 252-277.

14. Stanford JW, Weigel KV, Paffenbarger GC, Sweeney WT (1960) Compressive properties of hard tooth tissues and some restorative materials. J Am Dent Assoc 60, 746-756.

15. Wills DJ, Manderson RD (1977) Biomechanical aspects of the support of partial dentures. J Dent 5, 310-318

16. Caycik S, Jagger RG (1992) The effect of cross-linking chain length on mechanical proper- ties of a dough-molded poly (methylmethacrylate) resin. Dent Mater 8, 153-157.

17. Morris HF, Asgar K (1975) Physical properties and microstructure of four new commercial partial denture alloys. J Prosthet Dent 33, 36-46.

18. Niinomi M (1998) Mechanical properties of biomedical titanium alloys. Mater Sci Eng A Struct Mater 243, 231-236.

19. Muhlemann HR (1951) Periodontometry, a method for measuring tooth mobility. Oral Surg Oral Med Oral Pathol 4, 1220-1233.

20. Parfitt GJ (1960) Measurement of the physiological mobility of individual teeth in an axial direction. J Dent Res 39, 608-618.

21. Korioth TW, Hannam AG (1994) Deformation of the human mandible during simulated tooth clenching. J Dent Res 73, 56-66.

22. Ohyama T, Nakabayashi S, Yasuda H, Kase T, Namaki S (2020) Mechanical analysis of the effects of implant position and abutment height on implant-assisted removable partial dentures. J Prosthodont Res 64, 340-345.

23. Van Eijden TM (2000) Biomechanics of the mandible. Crit Rev Oral Biol Med 11, 123-136.

24. Reilly DT, Burstein AH (1975) The elastic and ultimate properties of compact bone tissue. J Biomech 8, 393-405.

25. Avery CM, Skidmore M, Peden A, Pan J (2011) Biomechanical study of a unilocking T-plate system for prophylactic internal fixation of the radial osteocutaneous donor site using the sheep tibia model. Oral Oncol 47, 268-273. 